\title{
Smoking during pregnancy: how reliable are maternal self reports in New Zealand?
}

\author{
R P K Ford, D M Tappin, P J Schluter, C J Wild
}

\begin{abstract}
Objective-To determine the reliability of self reports of smoking during pregnancy. Methods-Residual sera from early and late antenatal blood samples were tested for cotinine for all pregnancies over a six month period. Over an overlapping 12 month period, a postal questionnaire on smoking was also sent to all new mothers $(n=4857)$ when their baby was $4-8$ weeks old. Smoking status from obstetric booking notes was also obtained.

Results-The cotinine-validated smoking prevalence was $31.3 \%$ for the first trimester and $27.7 \%$ for the third trimester. Questionnaire self reported prevalences were $19.2 \%$ and $15.7 \%$ for the first and third trimesters respectively, and $18.9 \%$ for obstetric booking. Of cotinine-validated smokers, $22 \%$ denied smoking-self deceivers. Of mothers who replied to the questionnaire, a half appeared to systematically under report the amount they smoked.
\end{abstract}

Conclusions-Nearly a quarter of smoking pregnant women did not report smoking. Moreover, of those who did, the amount smoked was often under reported. This tendency to under report may rise as pressures to stop smoking increase. Accurate measures of smoking prevalence in pregnant women will require objective testing.

( $(\mathcal{F}$ Epidemiol Community Health 1996;51:246-251)

There is overwhelming evidence that smoking in pregnancy is harmful to both mother and child. This has led to an optimistic public health goal in New Zealand to reduce smoking in pregnancy from $33 \%$ in 1995 to a level of $15 \%$ or lower by the year $2000 .^{1}$

In the past, smoking rates have usually been measured by self reports through questionnaires and interviews. However, when smoking prevalence has been determined by more objective measures such as serum cotinine $^{23}$ or thiocyanate ${ }^{34}$ assay, considerable discrepancies have been uncovered. The levels of misclassification between self report and objective tests vary depending upon the population being studied. This phenomenon of smokers reporting their smoking status as nonsmoker has been given terms such as "self deception" 5 and "deceivers". 2 In the general population the level of this misclassification is in the order of $5-10 \%,{ }^{46}$ but is very much increased in situations where there has been a focus on smoke cessation. ${ }^{27}$
Through increasing public health attention given to reducing smoking in pregnancy, it is likely that the self deception rate for maternal self reports is higher than $5-10 \%$, and a level of $38 \%$ has been reported in one study. ${ }^{8}$ The rising awareness of the detrimental effects of smoking is likely to increase the pressure on mothers to overcome their nicotine addition. This in turn is likely to coerce them to give more optimistic self reports as an indication that they wish to do what is best for their baby. This effort to please can manifest itself in two ways: firstly, by smokers reporting themselves as non-smokers and secondly, by smokers under-reporting the amount of cigarettes actually smoked.

This paper describes the prevalence of smoking measured by both self reports and serum cotinine assay and then examines the discrepancies between them.

\section{Methods}

POPULATION

The population sampled comprised all pregnant women who had at least one antenatal blood test during the six-month period November 1993 to April 1994, who had residual serum available after the processing of the blood sample, and whose baby was identified from birth notifications in Canterbury. There were 892 eligible "early" antenatal samples. Subsequent (or "late") antenatal blood samples were collected from November 1993 through to July 1994 in order to have serum samples that had been taken throughout pregnancy $(n=$ 790).

"Early" antenatal samples were limited to the first trimester (less than 15 weeks' gestation) and "late" antenatal samples were limited to more than 28 weeks' gestation. These times were compared to the questionnaire questions on the amount smoked at the beginning of pregnancy and at the end of pregnancy.

\section{SERUM COLLECTION AND COTININE ASSAY}

Routine antenatal blood tests are collected from pregnant women in the first (early sample) and third (late sample) trimesters. Residual serum samples from these routine blood tests were collected from all medical laboratories in Christchurch. Serum was decanted into $1 \mathrm{ml}$ tubes with the laboratory identification number and stored at $-20^{\circ} \mathrm{C}$. Serum cotinine was measured using the STC (SolarCare Technologies, 1745 Eaton Ave, Bethlehem, Pennsylvania, USA) ELISA assay. Cotinine standard curves were run on each plate with the levels 
of $12.5,25,50$, and $500 \mathrm{ng} / \mathrm{ml}$. Plates were incubated for 20 minutes and read by an automated optical density reader. Cotinine levels were placed into three categories: non-smoker $<15 \mathrm{ng} / \mathrm{ml},{ }^{2}$ lighter smokers $15-100 \mathrm{ng} / \mathrm{ml}$, and heavier smokers $>100 \mathrm{ng} / \mathrm{ml}^{6}{ }^{6}$ The test has a reported specificity of $100 \%$ (there is virtually no natural cotinine, use of a nicotine patch indicates a smoker and passive smoking gives cotinine levels of less than $15 \mathrm{ng} / \mathrm{ml}^{6}$ ).

\section{PATIENT INFORMATION}

In order to determine the gestation at which serum samples were collected, samples were matched with notifications of birth in the Canterbury region for the 12 months January to December 1994. Birth notification information and laboratory request demographic data were matched on the basis of first name, surname, and address: a match between a serum sample and a birth notification confirmed a live birth. An unmatched sample could be due to a therapeutic abortion, a miscarriage, a change in name, a birth outside the region, or insufficient details available for matching. Information was also available for gestational age and birth weight of the baby. It was possible to match $67.3 \%$ of all samples. Finally, personal identifiers were removed from the data and the cotinine test results added to the database anonymously.

\section{QUESTIONNAIRE}

A questionnaire was sent out to all mothers on the birth notification list $(n=4857)$ when their baby was between 4-8 weeks old. A follow up letter was sent after four weeks if the questionnaire had not been returned. There were $3546(73.0 \%)$ mothers who responded to the questionnaire: 339 (7.0\%) were sent back "returned to sender (RTS)"; there was no reply from $972(20.0 \%)$. Of all mothers who were sent out a questionnaire, there were 1399 $(28.8 \%)$ for whom at least one blood sample was available for cotinine analysis. Because of the similarities between the RTS and "no reply" groups, they were combined in the analysis as non-responders.

The non-responder mothers were significantly younger (mean age 26.9 years, SD 5.9) than the responders (mean 29.5 years, $S D$ $5.1), z=9.25, p<0.001$. The babies of the nonresponders had a significantly lower birth

\section{KEY POINTS}

- Twenty two per cent of women biochemically confirmed as smoking during pregnancy denied smoking when asked about this while filling in a questionnaire.

- Many women who reported that they smoked under reported the amount.

- Given the sensitivity and stigma associated with smoking, objective measurements are necessary to determine accurately the smoking prevalence. weight (mean $3335 \mathrm{~g}$, SD 632) compared with the responders (mean $3427 \mathrm{~g}, \mathrm{SD} 592$ ), $\mathrm{z}=$ $4.34, \mathrm{p}<0.001$.

\section{OBSTETRIC RECORDS}

The reliability of smoking status recorded in the obstetric records was also examined. Reported smoking status was extracted from a $10 \%$ sample (all mothers giving birth in the month of July) and for all non-responders; 684 records were investigated with 599 having a valid smoking response.

\section{QUESTIONNAIRE RESPONSES AND BLOOD} SAMPLES

To verify that the collection of antenatal blood samples was not biased, the numbers of samples obtained for both the first and third trimesters were examined by type of questionnaire response: first trimester blood samples were available for $657(18.5 \%)$ of responders and 198 $(15.1 \%)$ of non-responders $\left(\chi^{2}=7.74, \mathrm{df}=1\right.$, $\mathrm{p}=0.005)$, and third trimester samples for 535 $(15.1 \%)$ and $217(16.6 \%)$ respectively $\left(\chi^{2}=\right.$ $1.57, \mathrm{df}=1, \mathrm{p}=0.21$ ).

This implies that responders had a greater representation than non-responders for the first trimester but they had equal representation in the third trimester. This first trimester discrepancy could be explained by the comparatively long duration between the blood sample collection and the questionnaire being sent out and hence questionnaire "non-responding" mothers were more likely to move from the study area and have pregnancies more likely not resulting in a live birth. Taking these considerations into account there appeared to be little overall systematic bias in the collection of blood samples.

\section{ANALYSIS}

The principle hypothesis examined was whether there is a discrepancy between self reported smoking and cotinine evidence of smoking status. For this investigation, the cotinine levels were assumed to be the true and correct measurement of smoking status.

As the sample numbers were sufficiently large, appealing to the central limit theorem, tests between means and proportions of two populations were made assuming normality; categorical comparisons were done using the usual Pearson's $\chi^{2}$ test; and, in the first instance, agreement on a particular question by the same group of individuals from two separate sources was quantified by determination of the kappa coefficient. ${ }^{9}$ Kappa represents the proportion of potential agreement beyond chance that was achieved by the study. Should the two measurements agree perfectly then the expected value of kappa equals one. However, should the two measurements agree only at the chance level then the expected value of kappa is zero.

Further investigation into measurement agreement was conducted through the fitting of independence, symmetry, and conditional symmetry models. ${ }^{10}$ The independence model 


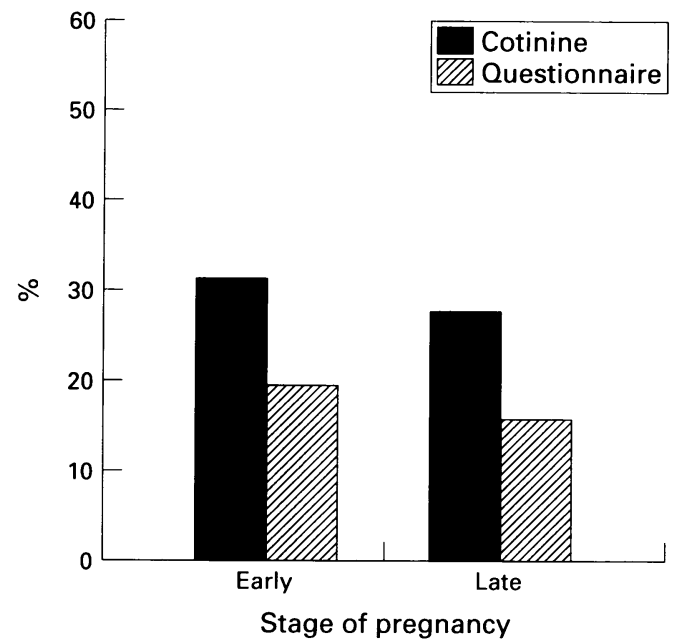

Figure 1 Smoking prevalence in relation to questionnaire estimate and cotinine assay for early (first trimester) and late (third trimester) pregnancy.

assumes that no relationship exists between smoking information sources beyond chance. The symmetry model, however, assumes that agreement exists between these smoking information sources and tests whether the numbers in cell $(i, j)$ equals the numbers in cell $(j$, $i)$ for all $i$ and $j$ cells of a table, $i$ not equal $j$ (ie: $n(i, j)=n(j, i))$. The conditional symmetry model, which also assumes agreement between smoking information sources, generalises the symmetry property by allowing either $\mathrm{n}(\mathrm{i}$, j) $>n(j, i)$ or $n(i, j)<n(j, i)$ for all $i<j$. That is, the conditional symmetry model allows greater numbers to appear above (or below) the main diagonal of a table compared to numbers below (or above).

The likelihood-ratio statistic $\left(\mathrm{G}^{2}\right)$ was used to test the fit of each model: the larger the value of $\mathrm{G}^{2}$, the more evidence there is against the model fitting the data. This likelihood-ratio was tested by approximating $\mathrm{G}^{2}$ to its associated chi-squared distribution and conducting the usual significance test.

This study was granted ethical approval from the Southern Regional Health Authority Ethics Committee.

\section{Results}

COMPARISONS OF SMOKING PREVALENCE FROM THE THREE DATA SOURCES

As the prevalence of smoking reportedly changes during pregnancy, data in the first instance were examined for the first (early samples) and the third (late samples) trimesters separately. Information on maternal smoking status was obtained from two independent sources, namely: serum cotinine assay; and self reports by questionnaire (fig 1 ). The smoking prevalences estimated by questionnaire were $19.2 \%$ and $15.7 \%$ for first and third trimesters respectively, while the cotinine-validated prevalences were $31.3 \%$ and $27.7 \%$ over those same stages of pregnancy. In addition, the questionnaire-estimated smoking prevalence before becoming pregnant was $31.1 \%$.

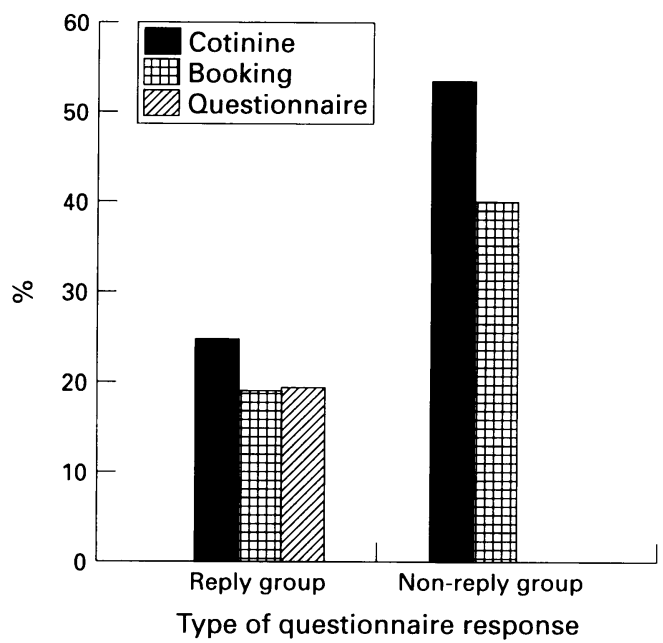

Figure 2 Smoking rates measured by questionnaire, booking information, and by cotinine concentration grouped by the type of questionnaire response (reply and non-reply).

Assuming that the objectively measured cotinine assay gives the true prevalence of maternal smoking, it is apparent from figure 1 that there is a considerable discrepancy between questionnaire self reported responses and the true prevalence during pregnancy. While the prevalence of smoking changed over pregnancy, the level of discrepancy remained constant, with a shortfall of $12.1 \%$ and $12.0 \%$ for first and third trimester questionnaire smoking responders compared to the corresponding cotinine measured prevalence $(\mathrm{z}=0.042, \mathrm{p}=$ $0.97)$. Although this level of misclassification remained static over pregnancy, the rate of misclassification increased. Based on the prevalence estimates contained in figure $1,38.7 \%$ of smoking mothers are not accounted for by questionnaire data in the first trimester compared with $43.3 \%$ in the last trimester.

To gain a further insight into the level of a smoking misclassification by questionnaire, smoking data were categorised by whether a reply to the postal questionnaire had been received: that is reply and no-reply. For those mothers replying to the questionnaire, smoking prevalence was estimated from three sources: serum cotinine assay, obstetric booking records, and from the postal questionnaire itself. However, obviously, only serum cotinine assay and obstetric booking information were available for the questionnaire non-reply mothers (fig 2).

Obstetric booking takes place in early pregnancy. Therefore, to make useful comparisons for this analysis, smoking prevalence was only determined from the first trimester questionnaire self reports and cotinine samples. In the "reply" group, the prevalences of self reported smoking were similar for both the questionnaire $(19.2 \% ; 95 \% \mathrm{CI}, 17.9,20.4)$ and booking records $(18.9 \% ; 95 \% \mathrm{CI}, 16.8$, 21.1). A high degree of agreement existed between results obtained from these two sources (kappa $0.71 ; 95 \% \mathrm{CI}, 0.59,0.84$ ), with $90.7 \%$ of mothers supplying concordant answers. 


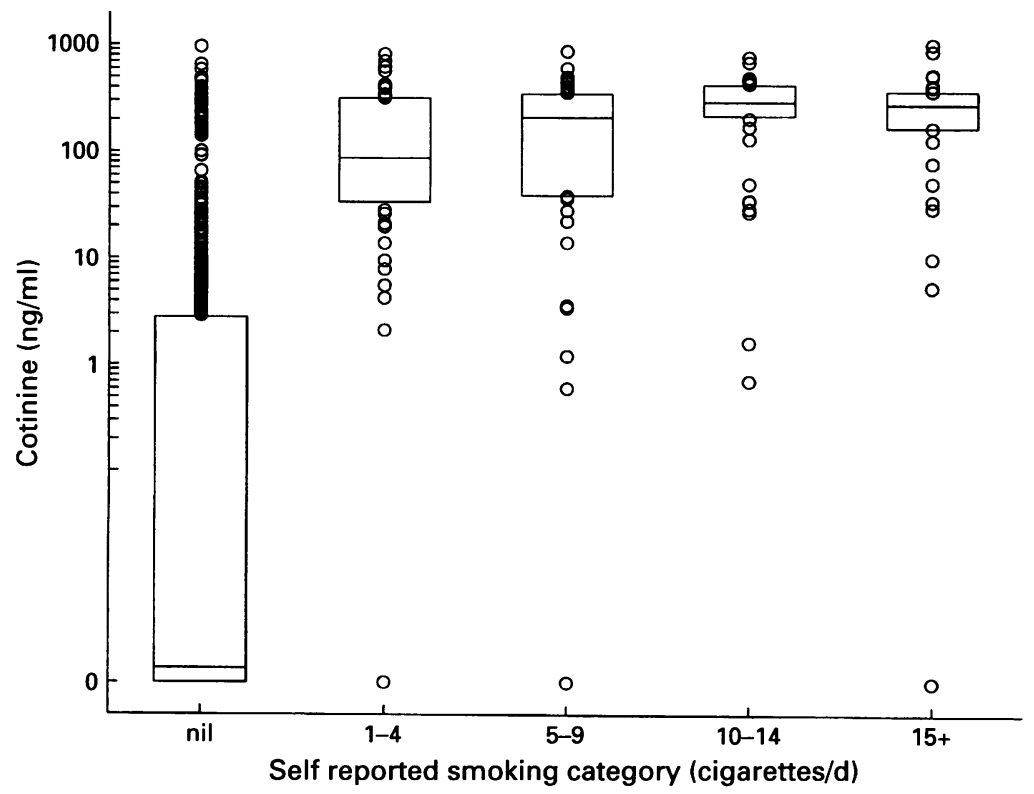

Figure 3 The distribution of cotinine measurements for each self reported category of smoking obtained from the questionnaire. The boxes enclose all values between the 25th and 75th centiles. The centre line corresponds to the median, the 50th centile. The circles represent individual cotinine values falling outside the boxes. $A$ log scale has been used to plot the cotinine values ( $\mathrm{ng} / \mathrm{ml}$ ).

Table 1 Cotinine categories compared with questionnaire self reported levels of smoking

\begin{tabular}{lllll}
\hline \multicolumn{4}{c}{ Cotinine determined categories of smoking } & \\
\cline { 2 - 4 } $\begin{array}{l}\text { Self reported smoking levels } \\
\text { (cig/d) }\end{array}$ & Nil & Lighter & Heavier & \\
& $(0-14 \mathrm{ng} / \mathrm{ml})$ & $(15-100 \mathrm{ng} / \mathrm{ml})$ & $(>100 \mathrm{ng} / \mathrm{ml})$ & \\
& No $(\%)$ & No $(\%)$ & No $(\%)$ & Total \\
\hline Nil & $422(78.6)$ & $16(3.0)$ & $14(2.6)$ & 452 \\
Light $(1-9)$ & $7(1.3)$ & $18(3.4)$ & $29(5.4)$ & 54 \\
Heavy $(\geq 10)$ & $1(0.2)$ & $3(0.6)$ & $27(5.0)$ & 31 \\
Total & 430 & 37 & 70 & 537 \\
\hline
\end{tabular}

SELF DECEPTION OF NOT SMOKING

The cotinine measurements indicated that the true smoking rate was significantly higher than both the questionnaire $(\mathrm{z}=3.15, \mathrm{p}<0.001)$ and the booking records $(z=1.77, p=0.038)$ indicated. Under the assumption that the cotinine measurement gave the true smoking prevalence (at $24.5 \%$ ) and by using self reported smoking prevalence (questionnaire and booking records) of approximately $19 \%$, it is likely that $22 \%$ of smoking mothers were claiming that they were non-smokers.

For those mothers who did not reply to the questionnaire, self reports on smoking were only available from the obstetric booking notes. Of these mothers, 39.9\% (95\% CI, 34.6, 45.3) identified themselves as smokers at booking while $53.5 \%(95 \% \mathrm{CI}, 46.6,60.5)$ proved to be smokers according to the cotinine tests $(\mathrm{z}=3.03, \mathrm{p}=0.001)$. Again, assuming cotinine assay provides the true smoking prevalence, of those mothers not replying to the questionnaire and smoking, $25 \%$ have misclassified themselves as non-smokers.

Overall, it is evident that the smoking prevalence for those replying to the questionnaire was considerably less than that in those not replying, regardless of the estimation sourcefrom cotinine tests, $24.5 \%$ v $53.5 \%(z=7.72$, $\mathrm{p}<0.001$ ); and from booking records, $18.9 \% \mathrm{v}$ $39.9 \%(z=5.35, p<0.001)$. However, about a quarter (22\% for repliers and $25 \%$ for nonrepliers) of smoking mothers reported themselves as non-smokers, regardless of whether or not they replied to the questionnaire. This is termed as self deception.

COMPARISONS BETWEEN COTININE LEVELS AND SELF REPORTED AMOUNTS SMOKED

The distribution of cotinine measurements for each self reported category of smoking, obtained from the questionnaire, is illustrated using a box-plot (fig 3). (The boxes enclose all values between the 25 th and 75 th centiles. The centre line corresponds to the median, the 50th centile. The circles indicate all other individual values.) The cotinine values are drawn on a log scale to reflect the accuracy of the assay at the higher values as the test was primarily designed to distinguish smokers from nonsmokers.

Based on figure 3, it can be observed that cotinine measurements fall naturally into three semiquantitative measurements for the amount smoked: nil (<15 ng/ml); lighter (15-100 ng/ $\mathrm{ml})$; and heavier (>100 $\mathrm{ng} / \mathrm{ml})$. These categories have been previously adopted by others. $^{26}$

Using cotinine as a semiquantitative measure, we examined smokers in the no-reply group to see if they were smoking more heavily than those in the reply group. Of mothers not replying to the questionnaire, $40.4 \%$ were classified as heavier smokers in the first trimester compared to only $16.3 \%$ of those who did reply $\left(\chi^{2}=51.8, \mathrm{df}=1, \mathrm{p}<0.001\right)$. A similar pattern emerged for third trimester comparisons.

\section{SELF DECEPTION OF AMOUNTS SMOKED}

Notice in figure 3 that there was little distinction between cotinine distributions for the two heaviest self reported smoking categories (ie: $10-14$ and $15+$ cigarettes/day). This suggests that a proportion of heavy smokers under reported the amount they actually smoked. We thus examined the degree of misclassification between self reported levels of smoking and their corresponding cotinine measurements (table 1). The comparison was made for mothers replying about their third trimester smoking as over this time the rate of deception amongst mothers appeared highest. Similar results were achieved when first trimester information was used.

For table 1, the sample kappa $=0.58$ (SE 0.03 ) providing strong evidence that agreement was better than if the categorisations were statistically independent. It appears that about half of mothers were under-reporting their smoking. To investigate the agreement structure more fully we fitted some statistical models.

As expected, the independence model fitted very poorly $\left(\mathrm{G}^{2}=580.6, \mathrm{df}=4\right)$. The symmetry model was considerably better $\left(\mathrm{G}^{2}=41.5, \mathrm{df}=\right.$ 3) but still provided a poor fit. However, the results for the conditional symmetry model $\left(\mathrm{G}^{2}=5.4, \mathrm{df}=2\right)$ were acceptable. Based upon this model for mothers having smoking cat- 
egories that were not in agreement (ie off the main diagonal), the probability that the self reported smoking was less than the cotinine categorisation was, on average, 5.36 times more likely than the reverse. This model provides strong evidence that mothers were systematically under-reporting the amount smoked.

\section{Discussion}

The purpose of this study was to determine the reliability of self reporting of smoking by mothers during their pregnancy. Self report data was based on obstetric booking records and on a postal self report questionnaire. Importantly, we were able to look at the smoking characteristics of those mothers who did not reply to the postal questionnaire through population cotinine testing and obstetric booking records. This combination of data enabled us to examine self deception in two ways: smokers claiming that they were non-smokers and professed smokers under-reporting the amount they actually smoked.

The cotinine validated prevalence of smoking in the first trimester was $31.3 \%$. However, if based on self report, the prevalence of smoking was found to be only $19.2 \%$. This is a very substantial discrepancy. Clearly, looking at self reports is not an accurate method of obtaining true prevalence figures for maternal smoking.

Although the response rate from the questionnaire was $73 \%$, only $43.3 \%$ of the cotininevalidated smokers responded: the no-reply group had a very much higher rate of smoking $(53.5 \%)$ in comparison to the reply group $(24.5 \%)$. From the combination of self report and cotinine testing we were able to calculate self deception rates of smokers. Surprisingly, the level of self deception was similar, at a level of around $25 \%$, in both reply and no-reply groups. This was despite the fact that there were many more heavier smokers in the noreply group $(40.4 \%)$ compared to the reply group $(16.3 \%)$. Our results are in the same order of magnitude of another study, ${ }^{8}$ which reported that as many as $38 \%$ of mothers who had a positive cotinine test reported themselves as not smoking.

We found that the level of self reported smoking given by questionnaire $(19.2 \%)$ was almost identical to that given at obstetric booking $(18.9 \%)$. The similarity between these two measurements may be an indication that the deception is a self deception rather than a conscious effort to mislead health workers. The questionnaire was anonymous and therefore gave the mother the opportunity to be honest without the fear of being admonished.

Of those mothers who did reply to the questionnaire, we uncovered a substantial degree of under reporting of amounts of cigarettes smoked. Of professed smokers, about half under reported their amount of smoking. This is also evident from figure 3, which shows in the two highest categories of self reported smoking that the cotinine values were similar. Those in the highest self reported smoking category (15 or more cigarettes per day) were likely to be giving bona fide answers, whilst the preceding category (10-14 cigarettes per day) was likely to include many under reporters.

The results of this study were from a population cohort based sample. The collection of antenatal blood samples for the cotinine analysis was a representative sample without any serious systematic bias. Serum cotinine assay has been reported as an accurate method of distinguishing smokers from non-smokers, with a sensitivity of $100 \%$ using a cut off measurement of $14 \mathrm{ng} / \mathrm{ml}^{2611}$ Cotinine has a $100 \%$ specificity-if there is cotinine detected in the blood it can only come from tobacco smoke or nicotine patches. Tobacco smoke exposure from passive smoking generally gives blood levels of less than $5 \mathrm{ng} / \mathrm{ml}$ and requires more sensitive methods of cotinine detection. If nicotine patches are being worn (although, not recommended in pregnancy) then this too is an indication for smoking.

The questionnaire estimated smoking prevalence before pregnancy was $31.1 \%$, which was strikingly similar to the cotinine-validated early pregnancy level of $31.3 \%$. So, it seems that once pregnant, many mothers become uncomfortable with the idea of smoking, leading to this phenomenon of self deception. This may indicate that the first step, a change in attitude, has been taken towards a change in smoking habit.

To grasp better the magnitude of the effect of self deception, we extrapolated our findings over the entire population of mothers under study $(n=4857)$ in the first trimester. From self report questionnaire information (smoking prevalence estimated at $19.2 \%$ ) the numbers of mothers smoking would be 930 (95\% CI, $870,990)$. But based on the cotinine figures (with repliers and non-repliers having smoking prevalences of $24.5 \%$ and $53.5 \%$, respectively) we estimate that there were actually $1570(95 \%$ CI, 1485, 1655) smoking mothers present in this population. Therefore, some 640 (41\%) smokers were not accounted for by the questionnaire. Finally, based on the self report prevalence of smoking gleaned from the obstetric data $(18.9 \%$ for repliers and $39.9 \%$ for nonrepliers), we estimate that of these 640 smoking mothers missed by the questionnaire, in 270 $(17 \%)$ it was due to not answering the questionnaire and in $370(23 \%)$ it was due to mothers' self deception.

The concept of self deception has been reported in a number of studies. Jarvis ${ }^{2}$ found that $19 \%$ of self reported non-smokers who had been questioned in an outpatient setting were in fact "deceivers" when cotinine levels were measured in their blood. In the CARDIA cohort study in the USA, there was a $4.2 \%$ misclassification between cotinine levels and self report, ${ }^{6}$ and they found that former smokers were much more likely to be misclassified. The amount of deception also appears to be influenced by the intensity of the anti-smoking environment-deception was more common in those who were trying to stop smoking. ${ }^{57}$ Therefore, it is likely that when more pressure is brought to bear on smokers, deception from self report data will increase. If this is the case, 
then repeated cross-sectional smoking studies based on self reports alone may show an improvement, not because of any real change in smoking status, but because of the increased denial of true smoker status following an increased awareness of its harm.

We conclude that prevalence measurements of smoking in pregnancy based on self report are very inaccurate. Obstetric booking data and self report from questionnaires give very similar results with a quarter of all smokers being self deceivers. We can therefore interpret this deception as a self deception rather than a deliberate attempt to mislead health workers. Not only do mothers deceive themselves about reporting their smoking but also substantially under report the amount they smoke. We expect, that as increasing pressure grows for women not to smoke during pregnancy, self deception rates will further increase. The only way to measure properly change in smoking prevalence during pregnancy is to use objective tests such as serum cotinine assay.

Funding: the blood sera collection, storage, and assay were funded by a New Zealand Asthma Foundation grant and Health Research Council of New Zealand grant. Drs Ford, Schluter, and Wild were funded, through employment, by
Healthlink South (which is a Crown Health Enterprise of New Zealand). Dr Tappin was funded by the Canterbury Cot Death

Fellowship.

1 Public Health Commission. Towards healthy lives for New Zealanders. Public Health Commission New Zealand, 1993.

2 Jarvis MJ, Tunstall-Pedoe H, Feyerabend C, Vesey C, Saloojee Y. Comparison of tests used to distinguish smokers from nonsmokers. Am $\mathcal{f}$ Public Health 1987;77:1435-8.

3 Haley NJ, Axelrad CM, Tilton KA. Validation of self-reported smoking behaviour: biochemical analyses of cotinine and thiocyanate. Am $\Im$ Public Health 1983;73:1204-7.

4 Kornitzer M, Vanhemeldonck A, Bourdoux P, de Backer G. Belgian heart disease prevention project: comparison of self-reported smoking behaviour with serum thiocyanate of self-reported smoking behaviour with serum thiocyanate
concentrations. F Epidemiology Community Health 1983; 37:132-6

5 Sillett RW, Wilson MD, Malcolm RE, Ball KP. Deception among smokers. BMF 1978;2:1185-6.

6 Wagenknecht LE, Burke GL, Perkins LL, Haley NJ, Friedman GD. Misclassification of smoking status in the CARDIA study: a comparison of self-report with serum cotinine levels. Am $\mathcal{F}$ Public Health 1992;82:33-6.

7 Kendrick JS, Zahniser C, Miller N, et al. Integrating smoking cessation into routine public prenatal care: the smoking cessation in pregnancy project. Am $\mathcal{F}$ Public Health 1995; 85:217-22.

8 Bardy AH, Seppala T, Lillsunde P, Kosela P, Gref CG. Objectively measured tobacco exposure among pregnant women in Finland in 1986 and 1990. Acta Obstet Gynecol Scand 1994;73:30-4.

9 Fleiss LJ. Statistical methods for rates and proportions. 2nd ed. Fleiss LJ. Statistical methods for rate

10 Agresti A. Categorical data analysis. New York: John Wiley, 1990.

11 Cummings SR, Richard RJ. Optimum cut off points for biomedical validation of smoking status. Am $\mathcal{f}$ Public Health 1988;78:574-5. 